\title{
Circulação colateral por vasa vasorum da artéria carótida interna ocluída - Breve relato de nove casos
}

\section{Report of Nine Cases of Collateralization of Occluded Internal Carotid Artery by Vasa Vasorum}

\author{
Rodrigo Menezes Xavier de Brito ${ }^{6}$ \\ ${ }^{1}$ Neurocirurgião do Hospital de Pronto Socorro João XXIII; \\ Neurorradiologista Intervencionista do Biocor Instituto, Hospital \\ Vila da Serra e Hospital Luxemburgo, Belo Horizonte, MG, Brasil \\ ${ }^{2}$ Neurorradiologista Intervencionista do Biocor Instituto e do Hospital \\ Vila da Serra, Luxemburgo e do Hospital Madre Teresa, Belo \\ Horizonte, MG, Brasil \\ ${ }^{3}$ Acadêmica do $6^{\circ}$ ano de Medicina da Faculdade da Saúde e Ecologia \\ Humana (FASEH), Vespasiano, MG, Brasil \\ ${ }^{4}$ Acadêmica do $6^{\circ}$ ano de Medicina da Faculdade de Medicina de \\ Barbacena, MG, Brasil \\ ${ }^{5}$ Neurorradilogista do Biocor Instituto e dos Hospitais Risoleta \\ Tolentino Neves, Vila da Serra, Luxemburgo e Madre Teresa, Belo \\ Horizonte, MG, Brasil \\ ${ }^{6}$ Neurologista do Hospital da Unimed, Belo Horizonte, MG, Brasil
}

Augusto César de Jesus Santos ${ }^{1}$ Rogério Zenóbio Darwich ${ }^{2}$ Ana Cláudia de Assis Capanema ${ }^{3}$ Fernanda Bretz Gomes dos Santos ${ }^{4}$ Ricardo Penteado Lana ${ }^{5}$ Romeu Vale Sant'anna ${ }^{6}$

\begin{abstract}
Address for correspondence Augusto César de Jesus Santos, MD, Rua Doutor Plínio de Morais, 788/101, Cidade Nova, Belo Horizonte, MG, Brazil CEP 31170-170 (e-mail: augusto992@msn.com).

Hospital Vila da Serra e Biocor Instituto, Belo Horizonte, MG, Brasil
\end{abstract}

Arq Bras Neurocir 2015;34:34-39.

\section{Resumo \\ Palavras-chave \\ - vasa vasorum \\ - artéria carótida interna \\ - circulação colateral \\ - neovascularização fisiológica \\ - estenose das carótidas \\ - angiografia}

\section{Abstract}

A recanalização da artéria carótida interna $(\mathrm{ACl})$ via vasa vasorum é um evento incomum. A confirmação de seu diagnóstico é dada pela arteriografia cerebral, que tem capacidade para a adequada identificação anatômica. A existência desse padrão de neovascularização está intimamente relacionada à obstrução carotídea por doença aterosclerótica. Relata-se série de nove casos com revascularização carotídea por vasa vasorum, com ênfase em sua angioarquitetura. A conduta foi conservadora em todos os pacientes. É de fundamental importância sua adequada e correta interpretação, para que não se promova intervenções desnecessárias.

The recanalization of the internal carotid artery (ICA) via vasa vasorum is an rare event. Cerebral arteriography is capable of identifying anatomically this problem and has been used to confirm its diagnosis. The existence of this neovascularization pattern is closely received

January 29, 2014

accepted

November 1, 2014
DOI http://dx.doi.org/ $10.1055 / \mathrm{s}-0035-1547387$ ISSN $0103-5355$.
Copyright $\odot 2015$ by Thieme Publicações License terms Ltda, Rio de Janeiro, Brazil
(1) $\Theta \circledast$ 


\section{Keywords}

- vasa vasorum

- carotid internal artery

- collateral circulation

- neovascularization physiologic

- carotid stenosis

- angiography related to carotid atherosclerotic obstructive disease. Series of 9 cases with carotid revascularization by vasa vasorum, with emphasis on its angioarchitecture were reported in this study. The approach was conservative in all patients. The proper and correct interpretation of the disease is very important to avoid unnecessary interventions.

\section{Introdução}

A recanalização da artéria carótida interna $(\mathrm{ACI})$ via vasa vasorum é um fenômeno raro. ${ }^{1-4} 0$ diagnóstico definitivo deve ser realizado pela arteriografia cerebral. Os métodos usados de rotina em triagens podem subestimar os casos de circulação colateral pela vasa vasorum da $\mathrm{ACI}^{2}$ Atualmente, estudos com o uso de angiotomografia ou angiografia por ressonância magnética aumentam as possibilidades de identificação desses achados. ${ }^{2}$

A vasa vasorum é responsável pela nutrição da túnica adventícia e parte da túnica médica da artéria carótida. Ela se origina diretamente do lúmen da $\mathrm{ACI}$, mas pode surgir

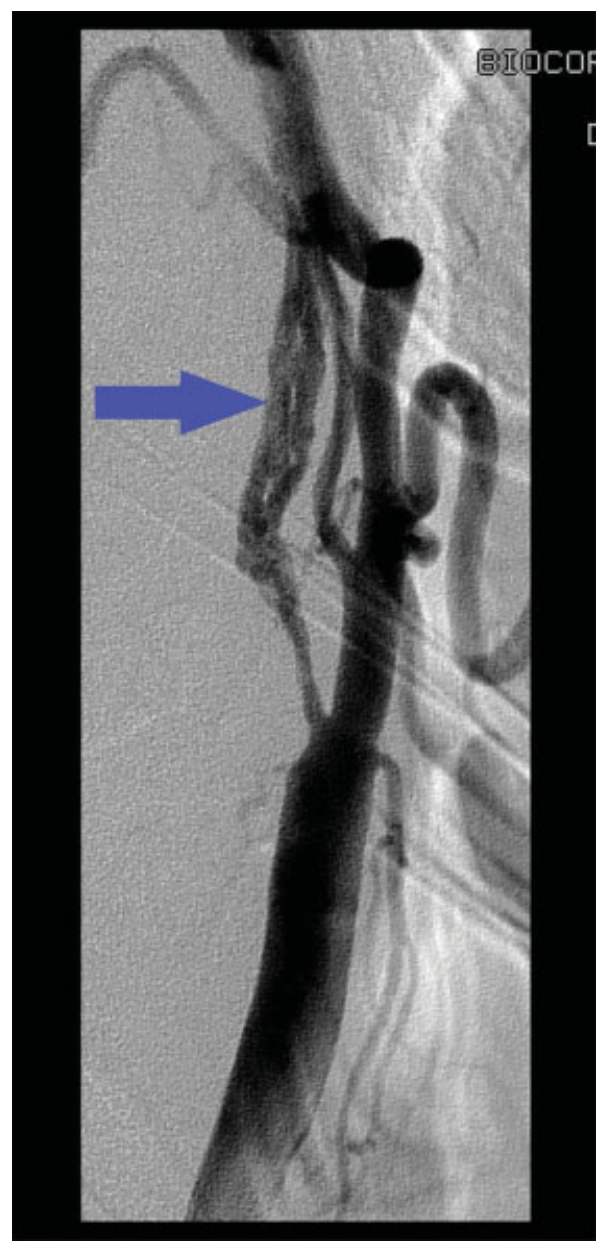

Fig. 1 Presença de vasa vasorum (seta) opacificando ACI. também das artérias tireóidea superior e faríngea ascendente e, nesses casos, participam no processo de revascularização via vasa vasorum. $^{5}$ Em estudo realizado em cadáveres, concluiu-se que esse padrão de neovascularização da ACI por vasa vasorum é mais prevalente através de túnica adventícia das artérias proximais como carótida interna, basilar e vertebral. ${ }^{6}$ A formação neovascular também pode ser encontrada através do interior da placa/trombo, assumindo aspecto menos tortuoso.

0 presente artigo demonstra o padrão de circulação colateral em doenças obstrutivas da artéria carótida interna através de anastomoses com a vasa vasorum e a contribuição do sistema faríngeo ascendente neste tipo de revascularização.

\section{Relato dos Casos}

Nove pacientes que apresentavam doença aterosclerótica que envolvia a bifurcação das artérias carótidas comuns receberam diagnóstico de oclusão da carótida interna.

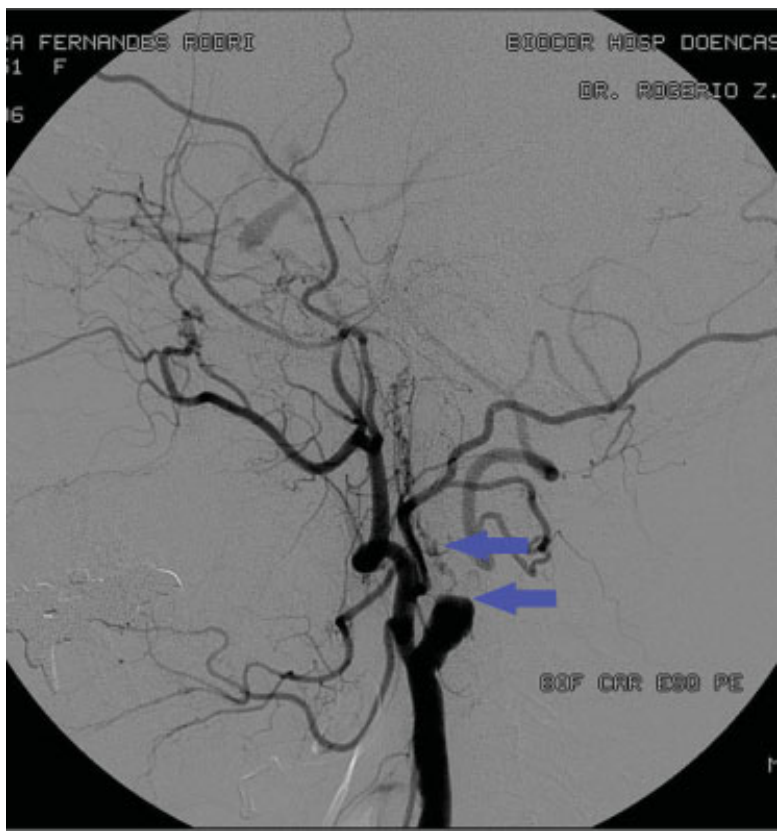

Fig. 2 Bifurcação carotídea esquerda revela carótida com fundo cego (seta inferior). À observação minuciosa pode-se notar a presença da vasa vasorum (seta superior). 


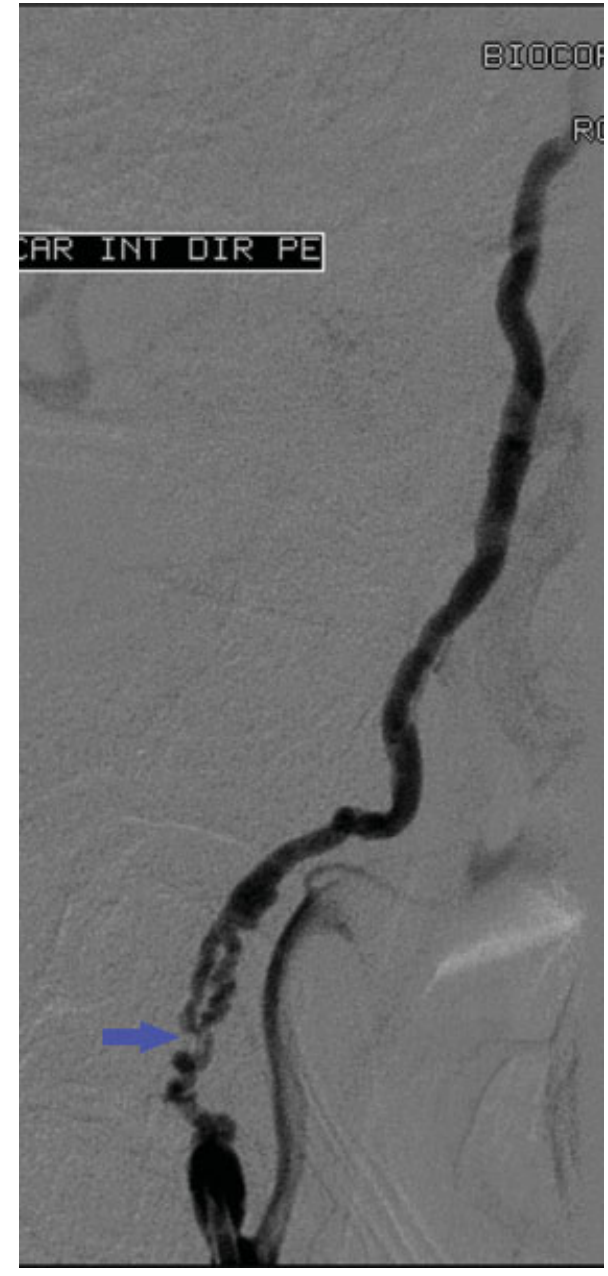

Fig. 3 A aproximação do cateter, após visualização de oclusão da $\mathrm{ACI}$ em uma primeira injeção de contraste, mostra a revascularização por vasa vasorum (seta).

Estes pacientes foram identificados em avaliação de ataque isquêmico transitório (AIT) ou através de triagem cardiovascular com doppler carotídeo, no Hospital Vila da Serra e no Biocor Instituto em Belo Horizonte/MG entre os anos 2000 e 2012. Destes pacientes, cinco pertenciam ao sexo feminino e quatro ao sexo masculino, com média de idade de 68 anos.

Sete pacientes apresentavam alterações neurológicas breves compatíveis com AIT. Foram, então, após avaliação inicial, submetidos à arteriografia cervicocraniana, através da qual se notou vasos serpiginosos múltiplos, configurando aspecto típico de neovascularização por vasa vasorum (-Figs. 1 a 7 ).

Um paciente, em investigação cardiovascular por doppler carotídeo, apresentava oclusão da ACI direita. No ano seguinte, novo doppler revelou a presença de fluxo no vaso. Seguiu-se investigação com angiorressonância que evidenciou um padrão atípico de circulação. Para esclarecimento, foi realizada arteriografia que mostrou o padrão de neovascularização por vasa vasorum (-Figs. 8A-C).

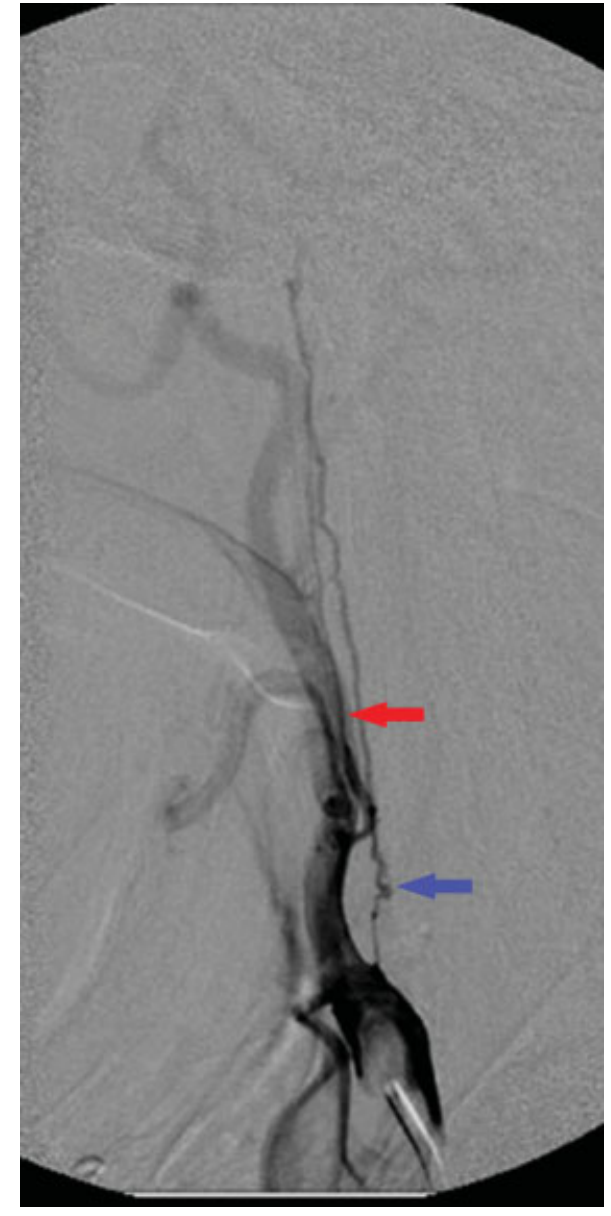

Fig. 4 A aproximação do cateter até a bifurcação carotídea revelou a existência de neovascularização por vasa vasorum (seta inferior) e pelo sistema faríngeo ascendente (seta superior).

Em um paciente portador de fibrilação atrial, em triagem cardiovascular, foi observado padrão de neovascularização por vasa vasorum sem lesões ateroscleróticas significativas nas carótidas ( - Fig. 9). Acredita-se que a obstrução carotídea seja decorrente de possíveis êmbolos de origem cardíaca.

Como este padrão de circulação colateral não acarreta riscos adicionais de novos eventos isquêmicos foi adotada a conduta conservadora em todos os casos.

\section{Discussão}

Circulação colateral por vasa vasorum da ACI ocluída é um fenômeno raro que ocorre mais comumente em pacientes com doença aterosclerótica avançada. ${ }^{3,4,6} \mathrm{~A}$ proliferação de vasa vasorum em placas ateromatosas está relacionada aos fatores angiogênicos e vascular para a íntima lesionada. Com a progressão da doença, com consequente oclusão carotídea, a vasa vasorum passa a atuar como um meio de revascularização. ${ }^{3,7}$

Aspectos serpiginosos e múltiplos desses pequenos vasos os diferem da suboclusão carotídea (string sign), que é 

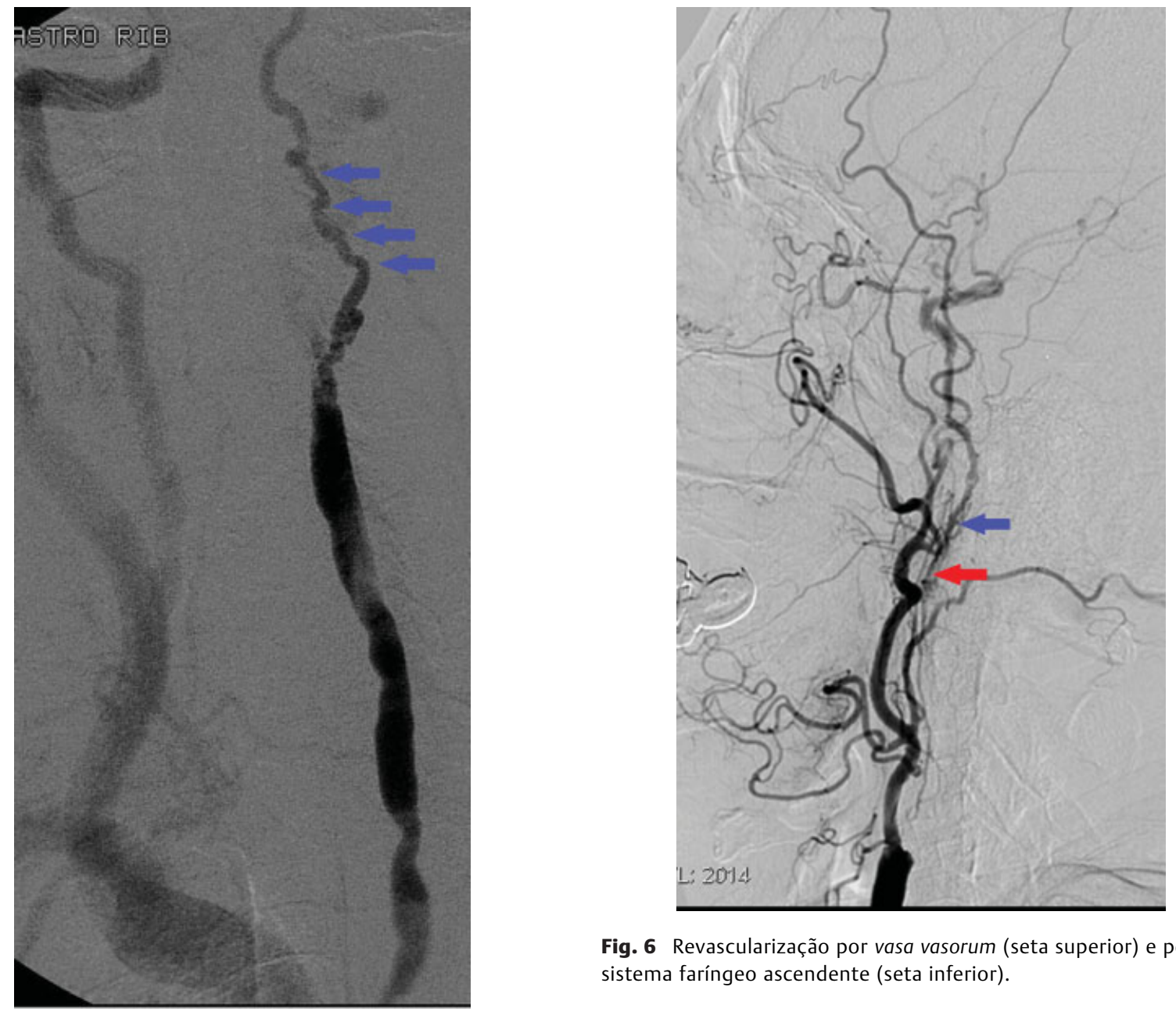

Fig. 6 Revascularização por vasa vasorum (seta superior) e pelo sistema faríngeo ascendente (seta inferior).

Fig. $5 \mathrm{ACl}$ com padrão vasa vasorum (setas).
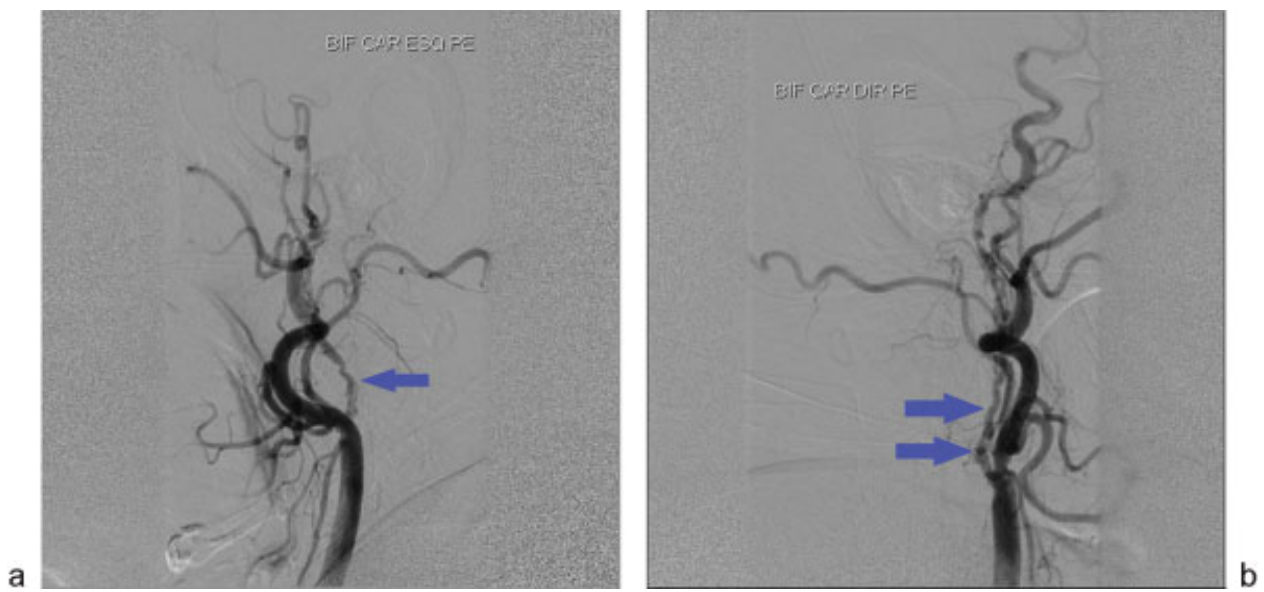

Fig. 7 Único caso com oclusão carotídea bilateral com o padrão vasa vasorum (indicado pelas setas). Pode-se observar em (a), a ACl direita; e em (b), a ACl esquerda. 

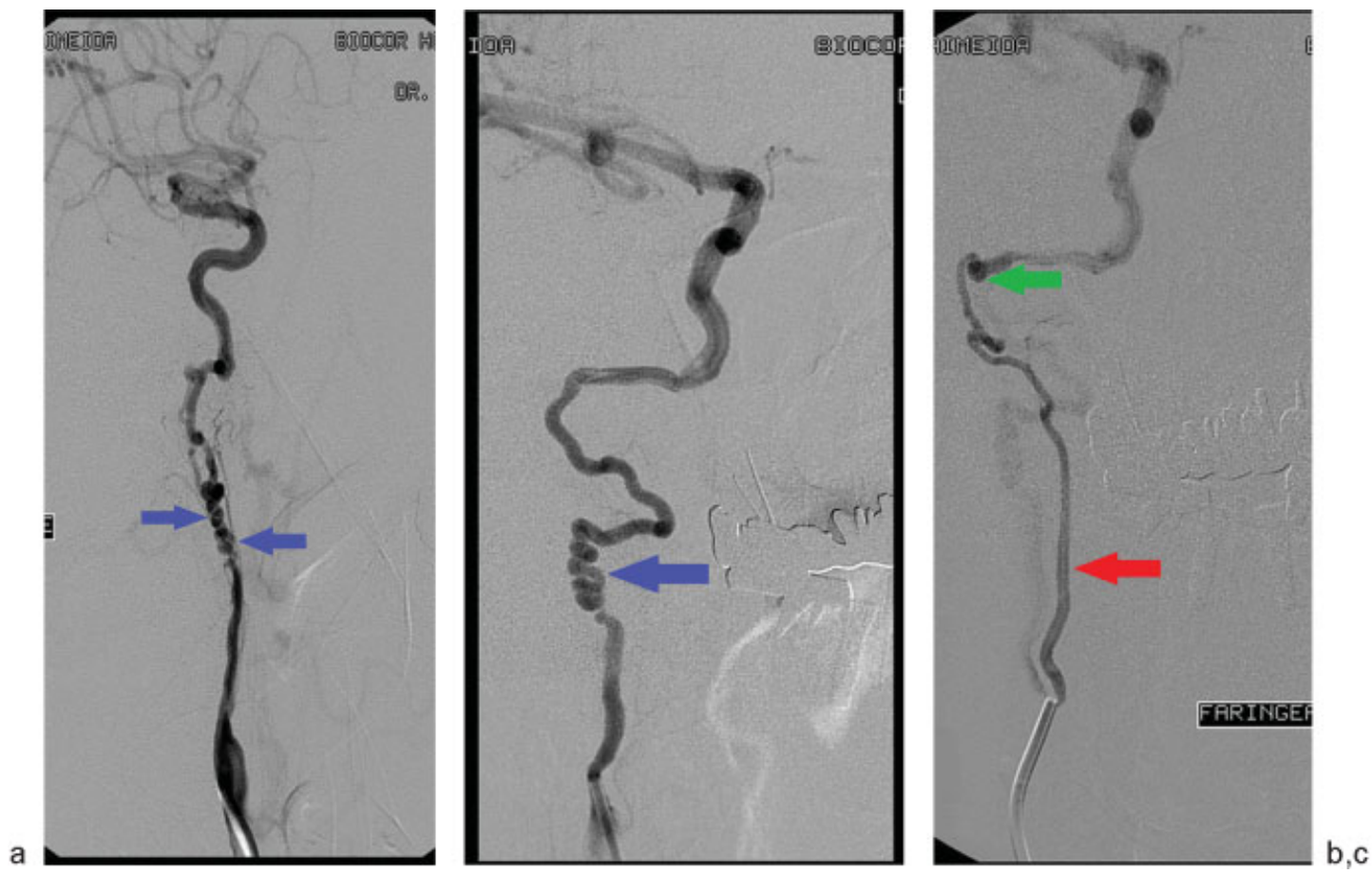

Fig. $8 \mathrm{Em}(\mathrm{a})$, nota-se a coexistência de dois sistemas vasculares atípicos (indicados pelas setas) que emergem da ACl ocluída. Em (b), seleção de um dos sistemas (vasa vasorum - seta). Em (c), seleção do outro sistema (artéria faríngea - seta inferior). Esses sistemas se unem formando a $\mathrm{ACl}$ intracraniana (seta superior).

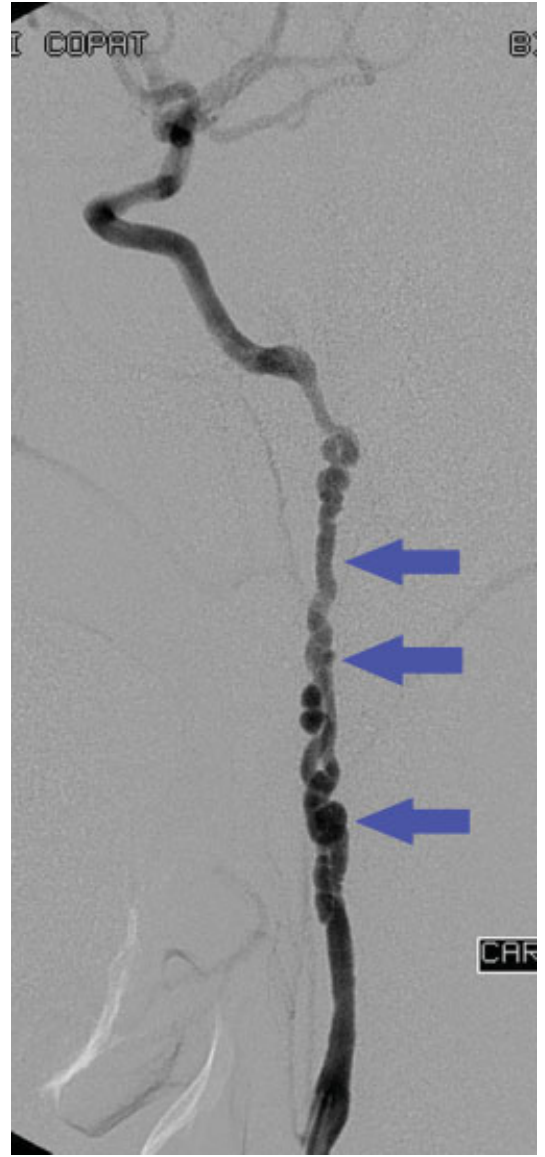

Fig. 9 Presença de neovascularização por vasa vasorum (indicado pelas setas). composto por filamento único e irregular, no mesmo trajeto da carótida interna. ${ }^{8}$

Takaba et al. ${ }^{6}$ identificaram circulação por vasa vasorum com maior prevalência em pacientes entre 60 e 69 anos.

Os nove casos relatados neste artigo apresentaram reestabelecimento da circulação carotídea por circulação colateral, distinguindo-os da doença suboclusiva da artéria carótida, principalmente na angioarquitetura. É importante esse diagnóstico diferencial para evitar tratamento cirúrgico desnecessário, já que não se encontra base científica para seu emprego neste tipo de revascularização.

\section{Conflitos de Interesse}

Os autores declaram não haver conflitos de interesse.

\section{Referências}

1 Klonaris C, Alexandrou A, Katsargyris A, Liasis N, Bastounis E. Late spontaneous recanalization of acute internal carotid artery occlusion. J Vasc Surg 2006;43(4):844-847

2 Nguyen-Huynh MN, Lev MH, Rordorf G. Spontaneous recanalization of internal carotid artery occlusion. Stroke 2003;34(4): $1032-1034$

3 Meguro T, Muraoka K, Terada K, Hirotsune N, Nishino S. Recanalisation of the internal carotid artery via the vasa vasorum after coil occlusion. Br J Radiol 2011;84(998):e23-e26

4 Baik SK, Park J, Kim GC, Lee TH, Kim YS. Vascular response to the vasa vasorum in a carotid artery dissecting aneurysm. Acta Neurochir (Wien) 2009;151(9):1159-1161 
5 Bo WJ, McKinney WM, Bowden RL. The origin and distribution of vasa vasorum at the bifurcation of the common carotid artery with atherosclerosis. Stroke 1989;20(11):1484-1487

6 Takaba M, Endo S, Kurimoto M, Kuwayama N, Nishijima M, Takaku A. Vasa vasorum of the intracranial arteries. Acta Neurochir (Wien) 1998;140(5):411-416
7 Colon GP, Deveikis JP, Dickinson LD. Revascularization of occluded internal carotid arteries by hypertrophied vasa vasorum: report of four cases. Neurosurgery 1999;45(3):634-637

8 Martin MA, Marotta TR. Vasa vasorum: another cause of the carotid string sign. AJNR Am J Neuroradiol 1999;20(2): 259-262 